\title{
Experiments on the flow past a circular cylinder at very high Reynolds number
}

\author{
By ANATOL ROSHKO \\ Guggenheim Aeronautical Laboratory, California Institute of Technology, \\ Pasadena, California
}

(Received 15 November 1960)

Measurements on a large circular cylinder in a pressurized wind tunnel at Reynolds numbers from $10^{6}$ to $10^{7}$ reveal a high Reynolds number transition in which the drag coefficient increases from its low supercritical value to a value 0.7 at $R=3.5 \times 10^{6}$ and then becomes constant. Also, for $R>3.5 \times 10^{6}$, definite vortex shedding occurs, with Strouhal number $0 \cdot 27$.

\section{Introduction}

Shortly after the closing and before the dismantling of the Southern California Co-operative Wind Tunnel (CW'T), some time was made available to us for a high Reynolds number experiment on a circular cylinder. In this large pressurized wind tunnel (Millikan 1957) it was possible to reach a cylinder Reynolds number $R$ of close to $10^{7}$, compared to about $2 \times 10^{6}$, the highest value for which wind tunnel measurements were previously reported in the literature. There are, in addition, some measurements in natural wind, up to about $R=4 \times 10^{6}$ (Dryden $\&$ Hill 1930; Pechstein 1940), about which we comment more fully later.

The many experimental measurements of drag coefficient $C_{d}$ at subcritical Reynolds numbers are in fairly good agreement as to the values of $C_{d}(R)$, but in the supercritical range, i.e. after the transition to low values of $C_{d}$, there is little agreement, except that $C_{d}$ lies between values of 0.2 and 0.4 . It is not clear whether the relatively large discrepancies here are due to difficulties in measurement or whether the flow here is more sensitive to the conditions of the experiment. The measurements of Delany \& Sorensen (1953) at Reynolds numbers up to $2 \times 10^{4}$ exhibit a multivaluedness which they attribute (private communication) to changes in the flow from a symmetrical to an unsymmetrical type, higher values of $C_{d}$ occurring in the unsymmetrical flow. Other authors claim to observe no asymmetries.

On the question of vortex shedding at high Reynolds number there is little information. It is well known that vortex shedding occurs at Reynolds numbers below the critical value, with a dimensionless frequency (Strouhal number $S$ ) of about $0 \cdot 2$. At critical and supercritical Reynolds numbers there are, to our knowledge, only two sets of measurements. The early ones by Relf \& Simmons (1924) indicate that in the transition range there is a predominant frequency in the wake, which they called 'aperiodic', compared to the 'accurately periodic' flow at $R<10^{5}$. Their measurements show that the Strouhal number of these frequencies increases as the drag coefficient $C_{d}$ decreases. 
More recently, Delany \& Sorensen (1953) obtained measurements at still higher Reynolds numbers $\left(10^{6}\right.$ to $\left.2 \times 10^{6}\right)$ using a pressure pick-up in the wake close behind the cylinder. The shedding frequencies, which were determined from the predominant frequencies on an oscillograph record, show considerable scatter, as do those of Relf \& Simmons; the values of $S$ are between 0.35 and 0.45 .

From these two sets of measurements, it would appear that $S$ rises rapidly in the interval $R=2 \times 10^{5}$ to $10^{6}$, and that there may be a rapid decrease at about $2 \times 10^{6}$.

Our intention in the present experiments was to overlap the Reynolds number range of the existing measurements, while extending them to values of $R$ as high as possible. The time available for preparing and performing the experiments was so short as to preclude a thorough investigation of all aspects of the flow, but it was hoped to obtain answers to a few obvious questions: Does the drag coefficient continue to change? Is there vortex shedding? Is there an asymptotic state, i.e. can we say anything about the ultimate form of the flow as $R \rightarrow \infty$, and at what values of $R$ are we approaching it?

\section{Experimental arrangement}

The experiments were performed in the subsonic test section of the CWT, which had a height of $8.5 \mathrm{ft}$. and width of $11 \mathrm{ft}$. It could be pressurized to $4 \mathrm{~atm}$; pressures of 1 and $2 \mathrm{~atm}$ were also used. To avoid compressibility effects, the flow speed was limited to a Mach number of about $0 \cdot 25$.

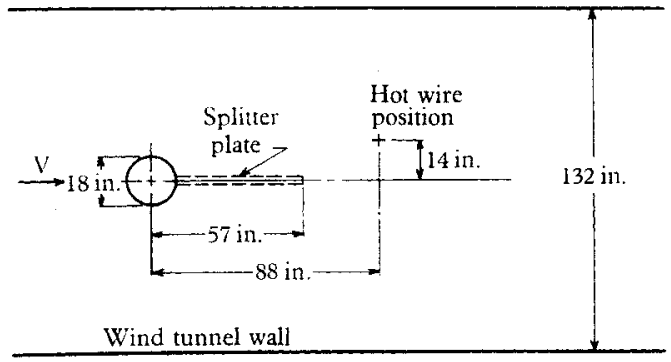

FrgURE I. Arrangement of cylinder in the wind tunnel.

The cylinder was a seamless 'black steel' pipe which had been sandblasted to remove its protective paint and scale. This resulted in a surface roughness of about $200 \mu$-in. The cylinder had a diameter of $18 \mathrm{in}$. and was round to within $\frac{1}{16}$ in. (in diameter). It spanned the $8 \frac{1}{2} \mathrm{ft}$. height of the test section. Pressure orifices were located every $10^{\circ}$ (with additional ones at $\theta=95^{\circ}$ and $105^{\circ}$ ), over half the circumference at the middle section. These were connected to a pressure measuring system consisting of pressure transducers which read out to digital indicators and recorders. The sensitivity of this system was set to give full output at the highest dynamic pressure, consequently there was a deterioration of accuracy at lower values of the dynamic pressure.

A hot-wire anemometer was mounted at a fixed position, $7 \cdot 3$ diameters downstream of the cylinder axis and 0.7 diameters off the centreline, as shown in figure 1 . The output of the hot wire, in a standard circuit, was fed into a spectral analyser, 
which could scan the frequency spectrum and record it on a pen recorder. We were looking for possible vortex shedding peaks in the spectrum. Whenever one was indicated on the record, it was tuned by hand and its frequency accurately determined by comparing it to an oscillator signal whose frequency was measured by an electronic counter.

Provision was made to install a 'splitter plate' on the centreline behind the cylinder, as indicated in figure 1. It also spanned the $8 \frac{1}{2} \mathrm{ft}$. height of the test section, extended $4 \mathrm{ft}$. along the centreline, and was $2 \mathrm{in}$. thick, being made up of two pieces of plywood bolted together.

\section{Wall interference corrections}

To obtain the highest possible Reynolds number, the cylinder diameter chosen was a little larger than might have been desirable from the point of view of the wall interference. With a diameter $d=1.5 \mathrm{ft}$. and tunnel breadth $b=11 \mathrm{ft}$., the blockage ratio was $d / h=0 \cdot 136$. To correct for the wall interference effects, we made use of the formulas of Allen \& Vincenti (1944), which give, for the corrected values of velocity and drag coefficient $V$ and $C_{d}$ in terms of the measured values $V^{\prime}$ and $C_{d}^{\prime}$,

$$
\begin{aligned}
& \frac{V}{V^{\prime}}=1+\frac{1}{4} C_{d}^{\prime}\left(\frac{d}{h}\right)+0 \cdot 82\left(\frac{d}{h}\right)^{2} \\
& \frac{C_{d}}{C_{d}^{\prime}}=1-\frac{1}{2} C_{d}^{\prime}\left(\frac{d}{h}\right)-2 \cdot 5\left(\frac{d}{h}\right)^{2}
\end{aligned}
$$

These formulas were obtained, following the earlier work of Lock, Glauert and Goldstein, by using image doublets to represent the interference between wall and cylinder, and image sources to represent the interference between wall and wake; the two effects give the third and second terms, respectively, in the formulas. Such an analysis does not take into account possible interference effects on the separation mechanism and the structure of the wake close behind the body; changes in these could have an important effect on the drag. This would be especially important in regions where $C_{d}$ is changing rapidly with $R$, but probably less important where $C_{d}$ is nearly constant. The experimental evidence relating to wall interference on cylinders is not entirely satisfactory. We have used equations (1) and (2) as the best available, believing them to be fairly accurate at our highest Reynolds numbers, where $C_{d}$ is nearly constant. The maximum corrections to $V^{\prime}$ and $C_{d}^{\prime}$ were about 4 and $10 \%$, respectively.

The uncorrected drag coefficient $C_{d}^{\prime}$ was obtained by integrating the measured pressure distribution $C_{p}^{\prime}(\theta)$; it was then corrected with equation (2). When corrected values of pressure coefficient $C_{p}$ were needed (e.g. figures 3 and 4), these were obtained from

$$
\left(C_{p}-1\right)=\left(\frac{V^{\prime}}{V}\right)^{2}\left(C_{p}^{\prime}-1\right)
$$

Corrections for Reynolds number $R$ and Strouhal number $S$ are the same as for the velocity. 


\section{Results}

\section{(a) Drag coefficient}

The corrected values $C_{d}$ are plotted against $R$ in figure 2. Also shown, for comparison, is the well-known curve of $C_{d}(R)$ at lower values of $R$. Different investigators do not agree exactly on this curve, but the differences are small, except in the supercritical region. We have chosen Wieselsberger's (1921) curve as representative and have also plotted the results of Delany \& Sorensen (1953) in the supercritical range (all the dashed lines). The latter extend to a higher Reynolds number than any other wind tunnel experiments of which we are aware, and they illustrate the difficulty in obtaining consistency in the supercritical range. It had been hoped that our experiments would overlap the

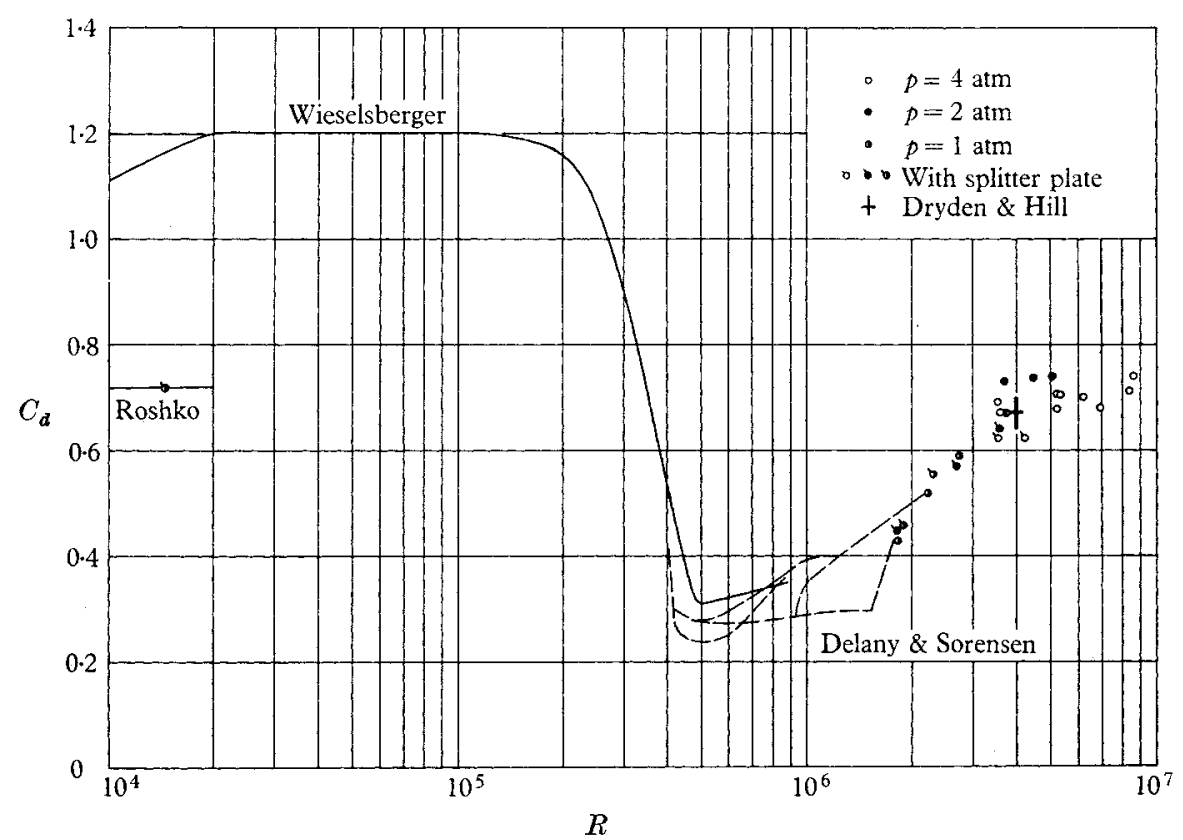

Figure 2. Drag coefficient.

existing measurements more than they do, but we were unable to obtain sufficient accuracy at our lowest Reynolds numbers, since the dynamic pressures were so low that the resolution of the pressure-measuring system became inadequate, and there was insufficient time to change it.

Our results match the trend of those at lower Reynolds numbers, as well as this can be determined from the multivalued behaviour, and it is apparent that $C_{d}$ increases in the range $10^{6}<R<3.5 \times 10^{6}$, from a value of about 0.3 to about $0 \cdot 7$, and then levels off at the latter value. It will be noted presently that the value $R=3.5 \times 10^{6}$ apparently marks the end of a transition range.

Also shown on figure 2 is the value of $C_{d}$ obtained by Dryden \& Hill (1930) in some experiments that are apparently not well known. These measurements were made on a smoke stack with a clear height of $120 \mathrm{ft}$., the values of $C_{d}$ being obtained from pressure distributions at a section $41 \mathrm{ft}$. from the top, where the 
diameter was $11 \cdot 8 \mathrm{ft}$. The wind speeds were about $25-40 \mathrm{~m} . \mathrm{p} . \mathrm{h}$., which corresponds to Reynolds numbers of 3-5 $510^{6}$. Their mean value of $C_{d}$ for a group of observations is $0 \cdot 67 \pm 0 \cdot 04$, and it may be seen that this agrees quite well with our results. A point of interest is that Dryden \& Hill inferred the wind speed from the measurements on the cylinder, making use of the fact that the maximum pressure is stagnation pressure, while the pressure at $31^{\circ}$ on either side of that point must be nearly static pressure. For experiments in the natural wind, this technique is clearly better than one using a velocity measurement at some location removed from the cylinder.

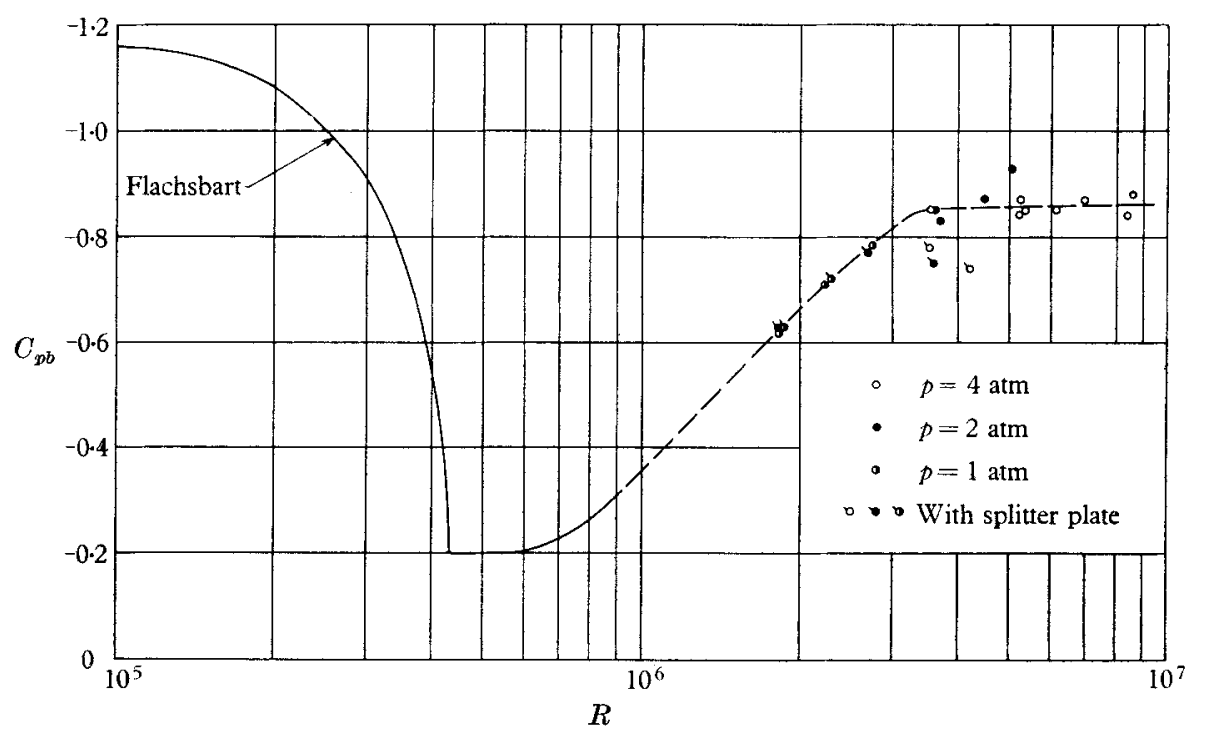

Figure 3. Base pressure coefficient.

In another set of experiments with a short stack $(l=30 \mathrm{ft} ., d=10 \mathrm{ft}$.), Dryden \& Hill obtained much lower values of $C_{d}$ (approximately $0 \cdot 4$ ) at the same values of $R$. The lower value may be attributed to the small length-diameter ratio, as may the similar results of Pechstein (1940) on a cylinder of length $10 \mathrm{~m}$ and diameter $2 \mathrm{~m}$ in a natural wind.

\section{(b) Base pressure coefficient}

Changes in $C_{d}$ are closely related to changes in $C_{p b}$, the pressure coefficient on the back of the cylinder. This was determined from the average pressure over 20 or $30^{\circ}$ on either side of the rearmost point. The results (corrected values) are plotted in figure 3, together with a curve obtained by Flachsbart (1929) at lower Reynolds numbers. The curve determined by our points is faired into Flachsbart's curve. (We have not included other investigators' results on this plot; inclusion of these would show a scatter or multivaluedness at supercritical Reynolds numbers, like that on the $C_{d}(R)$ curve.) 


\section{(c) Pressure distribution}

A pressure distribution at one of the highest Reynolds numbers $\left(8.4 \times 10^{6}\right)$ is shown in figure 4 , in which the corrected pressure coefficient $C_{p}$ is plotted against $\theta$, the angle measured from the stagnation point. Shown for comparison are typical pressure distributions at lower Reynolds numbers, $R=1 \cdot 1 \times 10^{5}$, which

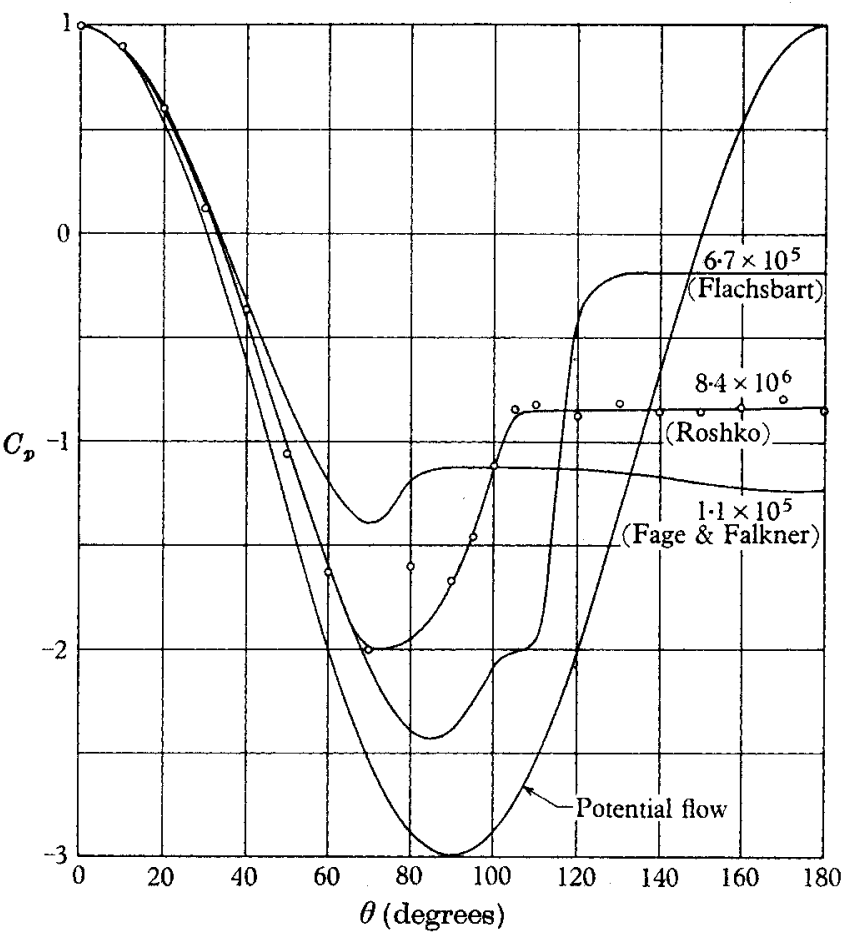

Figure 4. Pressure distributions.

is typical of subcritical Reynolds numbers, and $R=6.7 \times 10^{5}$, which is typical of Reynolds numbers just above the critical transition to low values of $C_{d}$. Our curve, which is nearly the same as the mean curve given by Dryden, differs from the other two. It shows a base pressure coefficient which is nearer to that of the subcritical distribution, but the distribution of pressure near $\theta=90^{\circ}$ indicates that the separation point is further back than in the subcritical flow, possibly at $90^{\circ}$ or greater. (The point at $\theta=80^{\circ}$ is a bad point; it has no significance with respect to separation.)

The pressure distributions for all our experiments are similar to this; the main effects of Reynolds number and splitter plate are on the base pressure coefficient (cf. figure 3).

\section{(d) Shedding frequency}

The Strouhal number $S$ of the vortex shedding frequency is plotted against $R$ in figure 5, together with previous measurements in the transition range and at subcritical Reynolds numbers. 
It should be pointed out that, with the technique used, the measurement of $S$ is much more accurate than that of $C_{d}$, since the accuracy does not decrease with decreasing dynamic pressure. This improved accuracy is apparent from the small scatter in $S(R)$.

Of much significance also is the fact that at our measuring station, vortex shedding was not observed at $R<3.5 \times 10^{6}$, although observations were made at all Reynolds numbers down to $0.9 \times 10^{6}$. The onset of shedding at $R=3.5 \times 10^{6}$ was quite definite. Below this value no peak frequency occurred; above this value there appeared a strong spectral peak, well above the turbulence level.

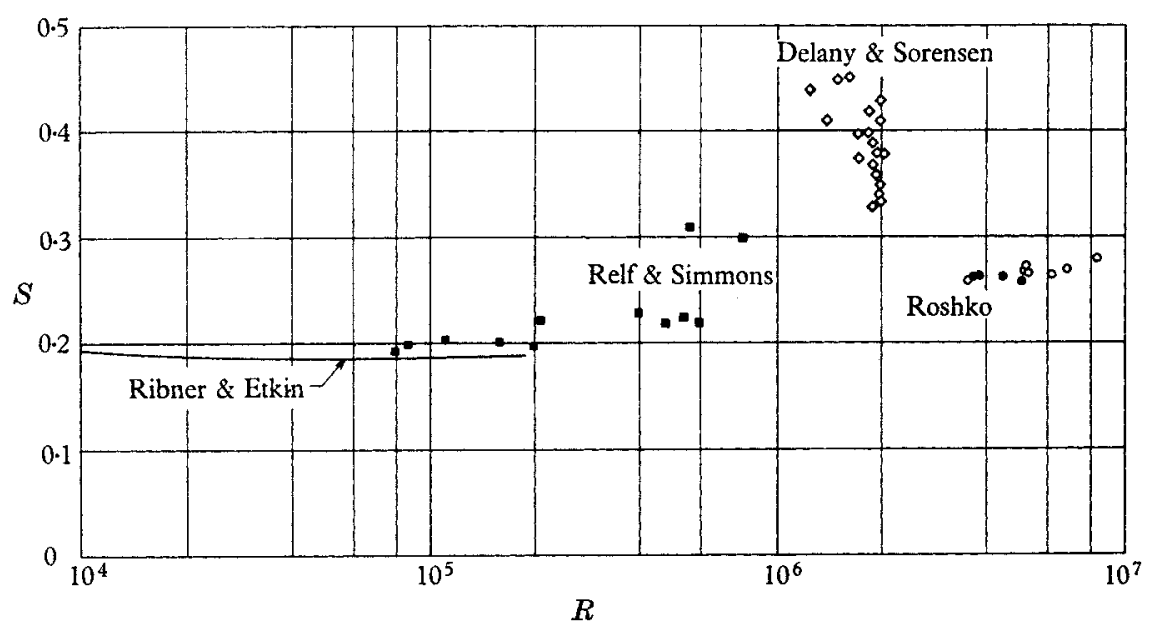

FIGURE 5. Strouhal number of vortex shedding frequency.

The (somewhat lower) harmonic (i.e. double the shedding frequency) also appeared, as is typical at subcritical Reynolds numbers for a hot wire not too far off the wake centreline. Although we were not able to make correlation measurements, say, at two wake positions, we have little doubt that what was observed was vortex shedding similar to that below $R=10^{5}$.

This raises the question of why we did not observe the frequencies reported by Delany \& Sorensen (1953) at values of $R$ between $10^{6}$ and $2 \times 10^{6}$. The latter measurements (as those of Relf \& Simmons 1924) were made with a probe only 1 or 2 diameters behind the cylinder, whereas ours was $7 \cdot 3$ diameters downstream of the cylinder axis. It was not possible to move it closer during the course of our experiments. We shall return to this point later.

Relf \& Simmons (1924) noted that an increase in shedding frequency is accompanied by a decrease in drag, and vice versa. This is illustrated in figure 6 , in which $C_{d}$ and $1 / S$ are plotted over a wide range of values of $R$.

\section{(e) Effect of the splitter plate}

Experimental points for which the splitter plate was installed behind the cylinder are indicated on the figures by flagged symbols. The main effects of the splitter plate are as follows. (i) The shedding is suppressed; there is no indication of a peak in the spectrum. (ii) The plot of $C_{d}$ (figure 2) indicates a small decrease 
in $C_{d}$ at values of $R$ above $3.5 \times 10^{6}$, but no effect at all at lower values. This is in contrast to the marked effect at subcritical Reynolds numbers observed in an earlier investigation (Roshko 1955). Evidence of a decrease is more obvious in figure 3, which clearly shows a decrease in $-C_{p b}$ at $R>3.5 \times 10^{6}$. (iii) There is no significant effect on the pressure distribution, other than that related to changes in the base pressure; the distributions are all similar to the example given in figure 4.

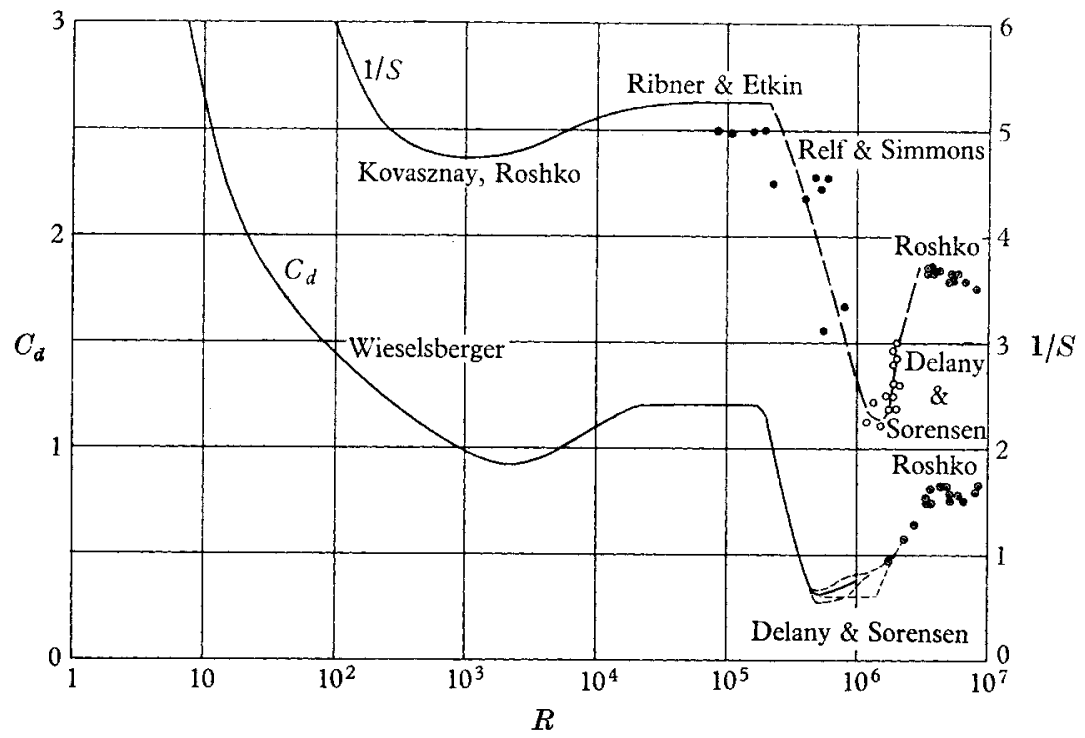

Figure 6. Drag coefficient and reciprocal of Strouhal number.

Unfortunately, it was not possible to go to Reynolds numbers as high as in the experiments without the splitter plate, because at the higher dynamic pressures severe flutter developed at the trailing edge of the splitter plate. The reason for this flutter is not clear; apparently it was not connected with vortex shedding off the cylinder, since this was suppressed by the plate. Possibly shedding off the trailing edge of the splitter plate itself had an effect.

\section{Ideas about the flow}

It was not possible in these experiments to make a detailed investigation of the wake structure, but from the results obtained we tentatively propose the following picture of the wake development.

\section{(a) Transitions and characteristic ranges}

The lower, or critical, transition at $2 \times 10^{5}<R<5 \times 10^{5}$, from high to low values of $C_{d}$, is followed by another (upper) transition, at $10^{6}<R<3.5 \times 10^{6}$, to a new plateau on which the coefficients have the following mean values: $C_{d}=0.70$, $C_{p b}=-0 \cdot 86, S=0 \cdot 267$. (Possibly the whole range from $R=2 \times 10^{5}$ to $3 \cdot 5 \times 10^{6}$ could simply be called the transition range.) For convenience in referring to them, we call the range on either side of the lower transition 'subcritical' 
$\left(C_{d}=1 \cdot 2\right)$ and 'supercritical' $\left(C_{d} \doteqdot 0 \cdot 3\right)$, respectively, according to established custom; let us call the plateau following the upper transition 'transcritical' $\left(C_{d}=0 \cdot 7\right)$.

As is well known, the (critical) Reynolds number at which the lower transition occurs can be lowered by introducing turbulence into the flow or roughness on the cylinder; we may expect a similar effect on the upper transition.

In the lower transition the wake width $d_{w}$ decreases from values larger than the cylinder diameter $d$ to values smaller than $d$, as the separation point moves from the front to the back of the cylinder. In the upper transition, the indication is that the wake 'opens' again, the separation point moving forward. However, it does not move to the front of the cylinder, and $d_{w}$ remains less than $d$. This view is supported by the form of the pressure distribution (figure 4) and by the values of $C_{p b}$ and $S$ at transcritical Reynolds numbers; the reasons are outlined in the next section.

A rapid opening of the wake would also explain the fact that, for the narrow wakes, we did not observe shedding frequencies 7 diameters behind the cylinder whereas Delany \& Sorenson did observe them close to the cylinder.

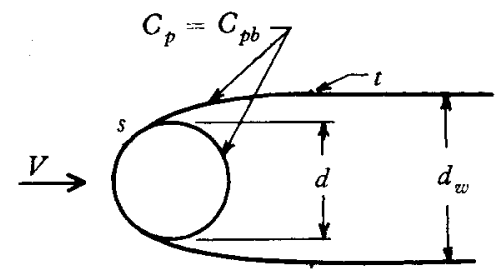

Type $\mathrm{A}\left(d_{w} / d>1\right)$

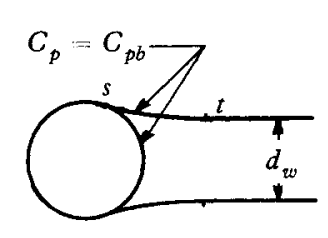

Type $\mathbf{B}\left(d_{w} / d<1\right)$

Frgure 7. Free-streamline model for relating base pressure coefficient to wake width.

\section{(b) Free-streamline model}

It has been found useful (Roshko 1954, 1955; Eppler 1954) to represent the mean flow in the vicinity of the cylinder by a free-streamline flow having the characteristics sketched in figure 7. In this model, the pressure on the wake boundary is constant $\left(C_{p}=C_{p b}\right)$ from the separation point $s$ to a point $t$ at which the boundary becomes parallel to the free stream. Beyond $t$ the wake width remains constant at the value $d_{w}$ while the pressure recovers to the free stream value. In a wake of type A, which is characteristic of the subcritical region, separation is on the front of the cylinder, and $d_{w} / d>1$; for type $\mathrm{B}$, which is characteristic of supercritical and transcritical flow, separation is on the back of the cylinder and $d_{w} / d<1$.

In some earlier work (Roshko 1954, 1955) we had postulated that the shedding frequency $n$ must depend only on the wake parameters $d_{w}$ and $V_{w}=V\left(1-C_{p w}\right)^{\frac{1}{2}}$, and that in terms of these there must be a universal Strouhal number, defined by $S^{*}=n d_{w} / V_{w}$, which is independent of the body producing the wake. Thus, it is related to the conventional Strouhal number $S=n d / V$ by

$$
\frac{S}{S^{*}}=\frac{d}{d_{w}} \frac{V_{w}}{V}=\frac{d}{d_{w}}\left(1-C_{p w}\right)^{\frac{1}{2}}
$$


From experimental determinations of $S$ and $C_{p w}$ for circular cylinders, wedges and flat plates at subcritical Reynolds numbers, and from the theoretical relation between $C_{p w}$ and $d_{w} / d$ for flows of type $A$, we concluded that the universal Strouhal number has the value $S^{*}=0 \cdot 16$.

Now if the nature of the vortex shedding at transcritical Reynolds numbers is basically the same as at subcritical values, then the universal Strouhal number should apply to this range also. We corroborate this as follows. It can be shown from a simple momentum consideration (Eppler 1954) that, for flows of the type shown in figure 7 ,

$$
C_{d}=\left(-C_{p s}\right)\left(d_{w} / d\right)
$$

Applying this formula to our measurements in the transcritical range (type $B$ ), we obtain $d_{w} / d=0 \cdot 70 /(0 \cdot 86)=0.81$. This value in equation (5) then gives $S=(0 \cdot 16)(1 \cdot 86)^{\frac{1}{2}} /(0 \cdot 81)=0 \cdot 27$, which checks very well with our mean experimental value $0 \cdot 267$.

These measurements seem to confirm, then, that at transcritical Reynolds numbers $d_{w} / d<1$, but the vortex shedding is otherwise similar to that at subcritical values.

Attempts to make similar calculations in the supercritical régime are unsuccessful. Taking experimental values of $C_{d}$ and $C_{p b}$ from several sources, we find that calculated values of $d_{w} / d$ vary from $0 \cdot 3$ to $1 \cdot 2$, the latter clearly in disagreement with observation, and calculated values of $S$ vary from $0 \cdot 15$ to $0 \cdot 7$. We must conclude that either the experimental data in this region is inaccurate, due to an inherent capriciousness of the flow, or the free-streamline model is inapplicable here.

\section{(c) Effect of the splitter plate}

In experiments at subcritical Reynolds numbers (Roshko 1954) it was found that a splitter plate has a strong influence on the flow. Briefly, the flow changes from one with alternate shedding to a steady symmetrical flow in which the separation streamline reattaches on the splitter plate, forming two closed recirculation regions on either side of the plate. At Reynolds numbers of the order of $10^{4}$, $C_{p b}$ changes from about -1.0 to -0.5 and $C_{d}$ from 1.2 to 0.7 (cf. figure 2 ).

The present experiments with a splitter plate at transcritical Reynolds numbers, while rather limited, due to the flutter problem mentioned earlier, also indicate a definite effect. The vortex shedding is stopped, $C_{p b}$ is changed from -0.86 to -0.76 , and $C_{d}$ is changed from 0.70 to 0.63 ; the changes in the coefficients are not nearly so spectacular as at subcritical Reynolds numbers.

In the upper transition leading to the transcritical régime, the splitter plate appears to produce no effect whatsoever on $C_{d}$ and $C_{p b}$. This raises the question as to whether, in the supercritical régime, there is true vortex shedding; interference with the alternating flow that must accompany vortex shedding should produce some effect.

In the experiments by Fung (1960) who measured the fluctuating forces on a cylinder at Reynolds numbers up to $1 \cdot 4 \times 10^{6}$, no definitely periodic force was observed, in contrast to the case at subcritical values (Ribner \& Etkin 1958). Unfortunately we were not able to look for the frequencies reported by Relf \& Simmons and Delany \& Sorenson and to observe the effect of the splitter plate 
on them, since we could not shift our hot-wire probe to a position near the cylinder.

\section{(d) Nature of the transitions}

The existence of the upper transition makes it somewhat difficult to interpret the flow in terms of the classical description of the critical Reynolds number, which is roughly as follows. 'At subcritical Reynolds numbers the separation is laminar, and occurs early, on the front of the cylinder. With increasing Reynolds number, transition in the boundary layer moves ahead of the laminar separation point, the now turbulent boundary layer can withstand a greater pressure rise, and so separation moves to the rear of the cylinder, with a consequent decrease in the drag coefficient.' To include the new results in this picture we would now have to say that in the upper transition a turbulent separation moves forward, finally reaching a stationary point, still on the back of the cylinder, and $C_{d}$ becomes constant.

It is probable that the actual events are somewhat more complicated. Bursnall \& Loftin (1951) have pointed out that in the supercritical range there is evidence of a laminar separation 'bubble', that is, a localized region consisting of a laminar separation, transition, reattachment and turbulent separation. Such bubbles have been observed to play an important role in the stalling of thin airfoils. The occurrence of a bubble is indicated, for instance, by a 'flat' in a rising pressure distribution, such as that at about $\theta=105^{\circ}$ in Flachsbart's curve (figure 4). If the region of localized separation is small, the flat may not be so definite. It is possible that a separation bubble occurs throughout the supercritical range. On the other hand, it is certain that at sufficiently high Reynolds number there will be no bubble. We suggest that the disappearance of a separation bubble characterizes the transition from the supercritical to the transcritical régime. (We are indebted to Dr I. Tani for a discussion of this point.) This suggestion is tentative, subject to further experimental investigation, but we have no other explanation of the upper transition.

Thus we would propose the following classification: at subcritical Reynolds numbers the separation is laminar, in the supercritical range there is a laminar separation bubble followed by turbulent separation, and in the transcritical range the separation is purely turbulent.

\section{Concluding remarks}

It is unfortunate that the time available for our experiments was too short to permit an investigation of some of the points raised in the preceding paragraphs, particularly since facilities for obtaining such high Reynolds numbers are not generally available. But even in the more accessible range of supercritical Reynolds numbers, additional work is needed. The question of vortex shedding in this range needs more attention and a study of the development of the laminar separation bubble would be useful.

We are left with the question concerning the asymptotic nature of the flow for $R \rightarrow \infty$. What would be the further effect of increasing $R$ beyond the values reached in our experiment? It seems unlikely that there will be any further transitions, since the point of boundary-layer transition can now only move 
smoothly forward on the cylinder. This may affect the location of the separation point, but can hardly be expected to produce rapid changes. There is then the question of Reynolds number effects on the flow in the wake. To detach this problem from the problem of separation it would be very useful to have a set of experiments on a flat plate normal to the flow over as wide a range of Reynolds numbers as we now have for the circular cylinder. For a plate (or for other sharp-edged configurations, such as wedges) the separation point is fixed at the edge, and changes in $C_{d}, C_{p b}$ and $S$ must be dependent only on the effect of $R$ on the wake mechanism. Such information should indicate the trend for $R \rightarrow \infty$.

We are indebted to the many people whose co-operation made this 'crash program' possible. The wind tunnel time was donated by the CWT owner companies, crews of the Guggenheim Aeronautical Laboratory and CWT assisted with the experiments, and the Fluid Mechanics group of the Jet Propulsion Laboratory provided instrumentation. David Russell and Dorothy Diamond assisted with the experiments and the computations, respectively.

\section{REFERENCES}

Aluen, H. J. \& Vincenti, W. G. 1944 Wall interference in a two-dimensional-flow wind tunnel, with consideration of the effect of compressibility. Nat. Adv. Comm. Aero., Wash., Rep. 782.

Bursnate, W. J. \& LofTin, L. K. 1951 Experimental investigation of localized regions of laminar boundary layer separation. Nat. Adv. Comm. Aero., Wash., Tech. Note 2338.

Detany, N. K. \& Sorensen, N. E. 1953 Low-speed drag of cylinders of various shapes. Nat. Adv. Comm. Aero., Wash., Tech. Note 3038.

Dryden, H. L. \& Hils, G. C. 1930 Wind pressure on circular cylinders and chimneys. Bur. Stand. J. Res., Wash., 5, 653-93.

EPPLeR, R. 1954 Beiträge zu Theorie und Anwendung der unstetigen Strömungen. $J$. Rational Tech. Anal. 3, 591-644.

Fage, A. \& Falkner, V. M. 1931 The flow around a circular cylinder. Aero. Res. Counc., Lond., Rep. and Mem. no. 1369.

Flachsbart, O. 1929 From an article by H. Muttray 1932. Handb. Experimentalphysik, 4, part 2 (Leipzig), 316.

FUNG, X. C. 1960 Fluctuating lift and drag acting on a cylinder in a flow at supercritical Reynolds numbers. J. Aero Space Sci. 27, 801-14.

KovasznaY, L. S. G. 1949 Hot-wire investigation of the wake behind cylinders at low Reynolds numbers. Proc. Roy. Soc. A, 198, 174-90.

MILLIKAN, C. B. 1957 Advanced education and academic research in aeronautics. $J . R$. Aero. Soc. 61, 793--808.

Pechstein, W. 1940 Der natürlichen Wind und seine Wirkung auf einen grösseren Kreiszylinder. Dissertation, Tech. Hochschule Hannover; reported by A. Pröll, 1942. Z. Ver. dtsch. Ing. 86, 222-3.

ReLF, E. F. \& Simmons, L. F. G. 1924 The frequency of eddies generated by the motion of circular cylinders through a fluid. Aero. Res. Counc., Lond., Rep. and Mem. no. 917.

Ribner, H. S. \& Erkin, B. 1958 Noise research in Canada. Proc. 1st Int. Congr. Aero. Sci., Madrid (publ. by Pergamon Press, London, 1959).

Roshko, A. 1954 On the drag and shedding frequency of bluff cylinders. Nat. Adv. Comm. Aero., Wash., Tech. Note 3169.

Roshko, A. 1955 On the wake and drag of bluff bodies. J. Aero. Sci. 22, 124-32.

Wieselsberger, C. 1921 Neuere Feststellungen über die Gesetze des Flüssigkeits- und Luftwiderstands. Phys. Z. 22, 321-8. 